(Aus der Universitäts-Frauenklinik Tübingen. [Direktor: Prof. Dr. A. Mayer].)

\title{
Ủber die Bedentung des Aneurysmas der Uteringefäße nach der Beobachtung eines Aneurysma arteriovenosum der Arteria und Vena uterina infolge Fliegerbombenverletzung.
}

\author{
Von \\ Priv.-Doz. Dr. Emil Vogt, \\ Oberarzt der Klinik. \\ Mit 4 Textabbildungen. \\ (Eingegangen am 3. Februar 1922.)
}

Die Lehre von den traumatischen Aneurysmen wurde durch das Massenexperiment des Krieges in riesigem Umfang erweitert und vertieft. Gegenüber den Aneurysmen der größeren und mittleren Gefäße an äußeren Körperteilen treten die Aneurysmen an Gefäßen der Brust-, Bauch- und Beckenhöhle sehr zurück, weil Verletzungen der Eingeweidearterien durch Verblutung sehr bald zum Tode führen oder unter der Diagnose innerer Blutung sofort operativ angegangen werden. Nach den Erfahrungen von Bier, Stich und Aschoff kamen im letzten Kriege traumatische Aneurysmen der Nieren, der Milz, des Magens, des Darms, der Blase und der Lunge nicht zur Beobachtung. Von früher her kennen wir traumatische Aneurysmen der Niere und der Leber.

Aneurysmen der Beckengefäße gehören zu den größten Seltenheiten. Aus der Friedensliteratur ist nur ein Fall bekannt. Mac Laren ${ }^{1}$ ) sah ein Aneurysma der Arteria iliaca interna im Anschluß an eine schwere Zangenentbindung. Es handelte sich um ein schräg verengtes Becken bei Sch wäche und Atrophie des ganzen linken Beines nach Kinderlähmung. Die Unterbindung proximal von der Gefäßgeschwulst brachte nur eine teilweise Heilung, die Pulsation war verringert.

Dieser Fall lehrt, daß das schräg verengte Becken bis zu einem gewissen Grade eine Kontraindikation für die Entbindung durch Zange darstellen kann. Wenigstens bei der atypischen und noch mehr bei der hohen Zange wird man recht vorsichtig zu Werke gehen müssen wegen der Gefahr der Druckschädigung der Nervenstämme und großen Blutgefäße auf der verengten Seite. Das alte Zangenmodell kann ja nur quer oder schräg zum Becken angelegt werden. Vielleicht bietet ihm 
gegenüber die Kiellandsche Zange einen Vorteil. Sie kommt ja bei jeder Art von Einstellung in den geraden Durchmesser des Beckens zu liegen.

Im Kriege wurden wenige Fälle von Aneurysmen der Beckengefäße bekannt. So berichtet Colmers $^{2}$ ) über ein Aneurysma der Arteria obturatoria nach Schußverletzung, welches durch Operation geheilt wurde, und ${ }^{\prime \prime}$ üttner ${ }^{3}$ ) über ein Aneurysma der Arteria hypogastrica, ebenfalls durch Schußverletzung entstanden. Ein Patient von Burkhardt kam nach Colmers durch Verblutung zum Exitus.

Über Aneurysmen der Uterusgefäße fand ich keine Angaben in der Literatur.

Unser Fall von Aneurysma arterio-venosum der Arteria und Vena uterina liegt folgendermaßen: Bei einem Fliegerangriff auf Tübingen am 12. 10. 1916 wurde die Frau durch Bombensplitter auf der Straße verletzt. Außer zahlreichen anderen, mehr oberflächlichen Verletzungen an den Extremitäten, am Rumpfe und am rechten Auge, hatte die Frau in der linken Gesäßhälfte einen talergroßen zerfetzten Einschuß. Die Wunde durchsetzte die Muskulatur, blutete aber nicht arteriell. 10 Wochen nach der Verletzung verließ Patientin die chirurgische Universitätsklinik, nachdem sämtliche Wunden unter konservativer Behandlung abgeheilt waren.

Im September d. J. wurde uns Patientin vom praktischen Arzt überwiesen wegen einer hartnäckigen Parametritis. Patientin gab an, daß sie seit der Verletzung sich nicht mehr erholt habe und viel weniger arbeitsfähig sei wie früher, ein starkes Ermüdungsgefühl beherrsche sie immer. Schmerzen im Unterleib, besonders auf der rechten Seite, von wechselnder Stärke würden sie bei Tag und Nacht nicht zur Ruhe kommen lassen. Die Periode sei regelmäßig, aber ziemlich stark.

Bei der Untersuchung der 40 jährigen Frau, die 3 gesunde Kinder geboren hat, war am auffallendsten der schlechte Ernährungszustand der Patientin, die Anämie und hochgradige Nervosität.

Gynäkologisch konnten wir den Befund einer erheblichen Parametritis bestätigen. Links vom Uterus fühlte man außerdem eine über hühnereigroße Schwellung, deren medialer Teil stark pulsierte und das vordere Scheidengewölbe halbkugelig vorwölbte, während der laterale Teil, welcher bis zur seitlichen Beckenwand reichte, sich mehr prall gespannt anfühlte. Beide Tumorabschnitte ließen sich ohne Schwierigkeit zusammendrücken, um sich mit dem Nachlassen des Druckes sofort wieder zu füllen. Die Pulsation in dem medialen Tumoranteil ging synchron mit dem Herzschlag. Außerdem war deutliches Schwirren und Reiben schon bei der vaginalen Untersuchung, noch viel stärker bei der rectalen Untersuchung zu fühlen. Der Versuch, die pulsatorischen Erscheinungen durch Druck auf die zuführende Arterie zum Schwinden zu bringen, war nicht ausführbar, weil der kurze, nicht veränderte $A b$ - 
nach der Beobachtung eines Aneurysma arteriovenosum der Arteria usw. 131

schnitt der Arteria uterina dicht an der Arteria hypogastrica nicht erreicht und zusammengedrückt werden konnte. Eine Differenz im Puls der rechten und linken Arteria femoralis war nicht vorhanden. Über dem oberflächlichen Verlauf der linken Arteria femoralis, der Arteria iliaca communis und unteren Aorta war ein dem Puls isochron gehendes, kontinuierlich schwirrendes Geräusch, welches sich bei der Systole verstärkte, mit dem Stethoskop deutlich zu hören. Nach der Feststellung, daß die Narbe eines größeren Einschusses im Gebiet des linken Glutaeus auf gleicher Höhe wie die fraglichen Tumoren liegt, bestand kein $Z_{\text {weifel }}$ mehr, daß es sich um ein traumatisches Aneurysma handelt.

Den Grundsatz der Chirurgie, jedes Aneurysma ähnlich wie eine maligne Neubildung zu betrachten und bald nach der Feststellung zu entfernen, mußte ich mir gerade auch unter dem frischen Eindruck meiner persönlichen kriegschirurgischen Erfahrungen im Felde für diesen Fall zu eigen machen. Mit der seltenen Spontanheilung durch Organisation und elastische Umwandlung der Wand, durch Thrombose und Thrombusorganisation dürfen wir nicht rechnen. Die meisten Aneurysmen neigen zum Fortschreiten und Wachstum, Verwachsungen mit den Nachbarorganen, selbst mit den Knochen sind die Folge. Die Nachbarorgane werden schließlich arrodiert oder komprimiert. Die Ruptur des Aneurysma kann jederzeit erfolgen und zu Verblutung nach außen oder innen führen, bevor Hilfe möglich ist. Die Gefahr der späteren sekundären Infektion durch Virulentwerden einer ruhenden Infektion ist ebenso wie die Möglichkeit einer hämatogenen Infektion nicht geringer einzuschätzen. Außerdem besteht stets die Gefahr einer Embolie.

In unserem speziellen Falle ergaben sich aus dem Sitz der Blutgefäßgeschwulst an den Uteringefäßen in der Tiefe des kleinen Beckens noch Besonderheiten, welche erst recht zur aktiven Behandlung drängten. Der linke Ureter hätte durch teilweise oder vollständige Kompression geschädigt werden können, die Möglichkeit einer Perforation in die blutgefäßreiche hintere Blasenwand lag nahe, ebenso wie die Gefahr der Druckschädigung der großen Nerven- und Gefäßstämme an der seitlichen Beckenwand. Der dauernde Wechsel des Blutgehaltes der Beckenorgane entsprechend der Menstruationswelle einerseits, andererseits die Lage des Aneurysmas zum Scheidengewölbe mit der Möglichkeit einer Verletzung und vaginalen oder parametranen Blutung wie zum Beispiel sub cohabitatione, das schwer zugängliche, in der Tiefe gelegene Operationsfeld bei einer dringlichen Operation, mit all diesen Überlegungen war die Indikation zu einer operativen Behandlung klar gegeben.

Wir sehen dabei ab von der Größe und Schwere der Unsumme von Gefahren, welche die Aneurysmaträgerin laufen müßte bei einer etwaigen Schwangerschaft, bei der Geburt und dem Wochenbett. Bei der natür- 
lichen, noch mehr bei des künstlichen Entbindung wäre eine Kompression des Aneurysmas und damit eine indirekte Verletzung des Aneurysmas unvermeidbar. Die Gefahr der direkten Verletzung würde sich steigern bei jedem künstlichen Eingriff, wie z. B. beim Einstellen des Muttermundes, beim Sprengen der Blase, bei der Naht eines Cervixrisses, bei der Tamponade, Austastung usw. Mit Beendigung der Geburt ist durchaus nicht jede Gefahr beseitigt. Im Wochenbett bieten sich für das Zustandekommen einer Infektion so zahlreiche Gelegenheiten, daß diese Komplikation und damit der Ausbruch einer Bakteriämie oder einer Pyämie die Katastrophe sehr leicht herbeiführen könnte. Auch diese Gefahren, welche das Fortpflanzungsgeschäft mit sich bringt, indizierten die sofortige Operation.

Vor der Laparotomie wurde noch die normale Tätigkeit des linken Ureters cystoskopisch festgestellt. Wegen der Gefahr des Aufflackerns einer ruhenden Tetanusinfektion wurde prophylaktisch Tetanusantitoxin eingespritzt.

In Lumbalanästhesie wurde nach Fascienquerschnitt die Radikaloperation ausgeführt. Die linke Arteria hypogastrica wurde prophylaktisch mit einem Faden umschlungen, um jederzeit blutleer operieren zu können. Der linke Ureter wurde in seinem ganzen pelvinen Verlaufe von demselben Peritonealschlitze aus freigelegt. Die Arteria uterina wurde dicht am Abgang aus der Arteria hypogastrica unterbunden. Zur Unterbindung nehmen wir selbstbereitetes Jodeatgut. Mit diesem Material versorgen wir schon eine Reihe von Jahren bei sämtlichen Operationen die Gefäße. Nachblutungen, gerade aus der Arteria uterina, welche von mancher Seite so gefürchtet werden, haben wir dabei nie erlebt. Das stimmt auch überein mit den Erfahrungen anderer Gynäkologen und den wissenschaftlichen Ausführungen von $v$. Gaza $a^{5}$ ). Die Operation verlief blutleer. Um eine Infektion zu bekämpfen, wurden $40 \mathrm{ccm}$ Preglsche Jodlösung vor Schluß der Bauchhöhle in das kleine Becken eingegossen, der Douglas wurde nach der Scheide zu drainiert. Die Frau überstand den Eingriff gut. Die Rekonvaleszenz war durch eine Parametritis kompliziert.

Das Präparat stellt den faustgroßen Uterus und die linken Adnexe dar, die rechten wurden aus technischen Gründen während der Operation abgetragen. Die linken Adnexe weisen makroskopisch keine gröberen Veränderungen auf. Die Gefäßgeschwulst besteht makroskopisch (Abb. 1) aus 2 Teilen: einem medialen, mehr ovalen in Pflaumengröße und einem lateralen, fast rundlichen, kastaniengroßen Abschnitte. Auf dem medialen sitzen Reste von derbfaserigem Bindegewebe und mehrere Gefäßstïmpfe fest.

Nach der Härtung in Formalin haben wir das Präparat geröntgt und fanden einen größeren Bombensplitter im Uterus und einen in nächster 
Nachbarschaft des Aneurysma (Abb. 2). Der im Uterus sitzende Splitter ist ziemlich groß. Der kleinere Splitter, welcher in der Nische zwischen den beiden Säcken des Aneurysmas liegt, ist sehr scharfkantig. Entweder kamen beide Splitter, außerhalb des Körpers getrennt, durch den gemeinsamen Einschuß in die Tiefe des Beckens, oder aber erst innerhalb des Körpers im Verlaufe des Schußkanales fand eine Teilung eines ursprünglich größeren Geschoßteiless statt. Solche Beobachtungen sind ja im Kriege öfters gemacht wordcn.

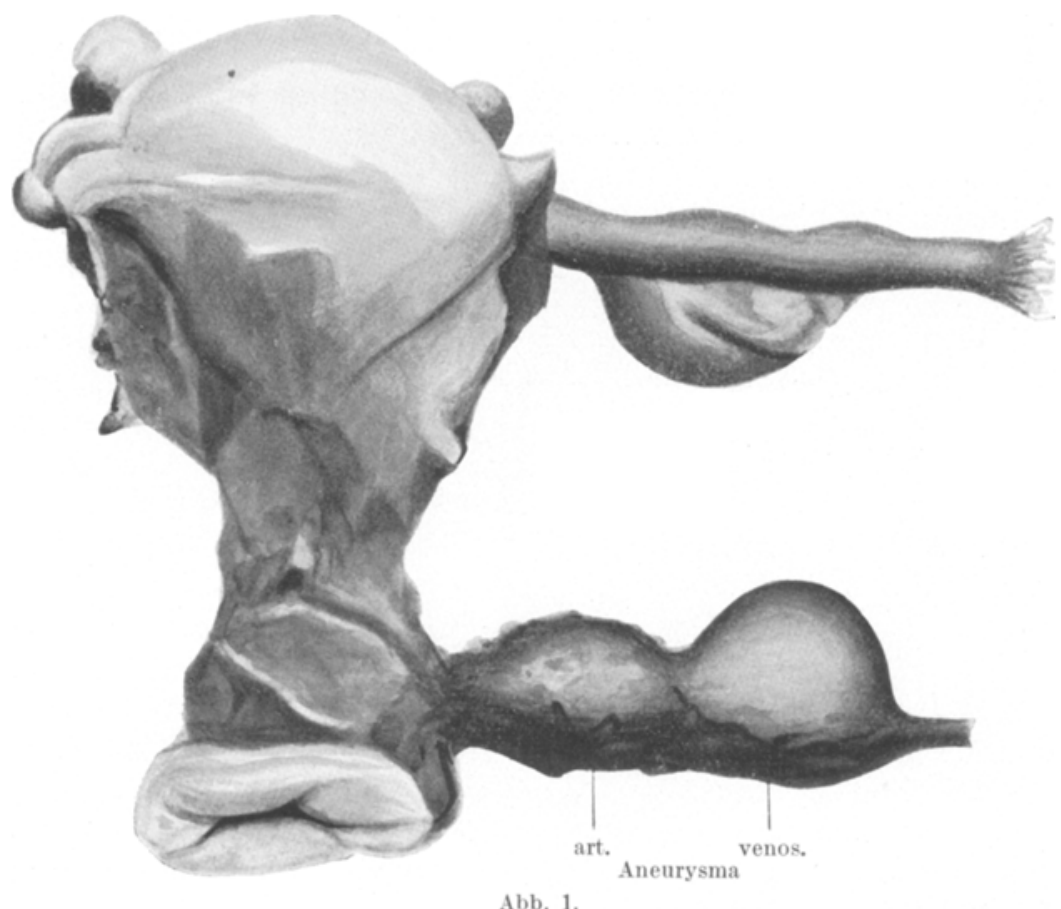

Aut einem Horizontalschnitte durch die Tumoren wurde gerade der kleinere Splitter mitgetroffen (Abb. 3). Dabei zeigte sich, daß wir es mit einem Aneurysma arteriovenosum traumaticum zu tun haben. Der mediale Teil ist das Aneurysma arteriosum und der laterale Teil das A. venosum. So erklärt sich jetzt der klinische Befund. 'Die mediale Geschwulst pulsierte mächtig mit fühlbarem Schwirren und Reiben, die laterale aber nicht, sie war nur prall elastisch und offenbar mit Flüssigkeit gefüllt. Beide Abschnitte waren aber in gleicher Weise zusammendrückbar.

Die Arterie und Vene sind augenscheinlich gleichzeitig verletzt worden. Nach der pathologisch-anatomischen Literatur bezeichnet man diese Formen des Aneurysmas als Varix aneurysmaticus oder nach der chirur- 
gischen Literatur entsprechend dem Vorschlage von Subbotitsch ${ }^{4}$ ) in neuerer Zeit als Aneurysma arteriovenosum directum mit Aneurysma arteriale und Varixbildung, oder noch viel einfacher nach $O$. Hahn als arteriovenöse Fistel.

Diese Form des Aneurysmas ist außerordentlich häufig, sie überwiegt bei weitem. Bier sah nach den Angaben von Stich 56 arteriovenöse und 45 arterielle Aneurysmen, Küttner $45 \%$ arterielle und $55 \%$ arteriovenöse Aneurysmen. In der großen Statistik von Räschke mit 1600 Fällen waren $57,9 \%$ arterielle und $42,1 \%$ arteriovenöse Aneurysmen verzeichnet.

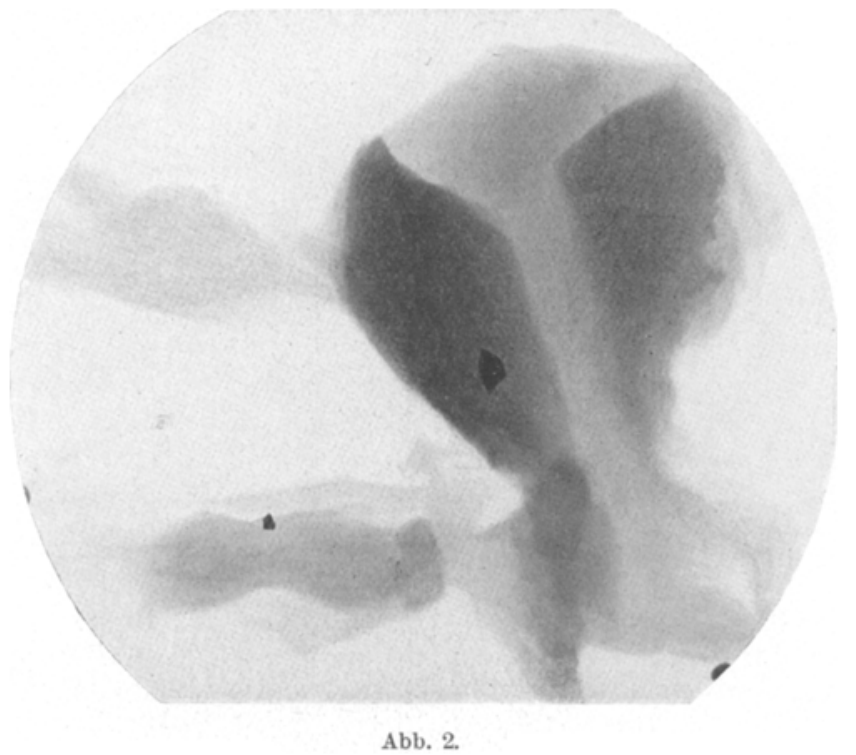

Beim Aneurysma arteriovenosum wieder kommt zuerst die direkte Form und dann die indirekte. So waren nach einer Statistik von Salomon $80 \%$ der Aneurysmen direkte Aneurysmen gegenüber nur $20 \%$ indirekten.

Die Richtigkeit der Diagnose eines Aneurysma arteriovenosum der Arteria und Vena uterina läßt sich aus dem klinischen Bilde und dem anatomischen Befunde beweisen. Die 3 klinischen Kardinalsymptome des Aneurysma arteriovenosum sind vorhanden. Es wurde durch die Palpation ein Tumor, die Pulsation des Tumors sowie ein Geräusch beim Betasten und Behorchen festgestellt. Die Größe des Tumors läßt sich sehr gut für das Aneurysma arteriovenosum verwerten. Bei dieser Form halten sich die Gefäßgeschwülste stets in mäßigen Grenzen und erreichen höchstens Hühnereigröße, sie zeigen keine Neigung zum Wachstum im Gegensatz zum reinen Aneurysma arteriale. Die Pulsation, das Schwirren und Reiben war ohne jede Schwierigkeit nachweisbar. Das 
Geräusch war über der Femoralis und der Tliaca communis bis zur Aorta herauf andauernd zu hören. Es verstärkte sich systolisch und wurde zentripetal am lautesten fortgeleitet, weniger zentrifugal. Nach dem Wahlschen Gesetz müßte es sich schon deshalb um ein Aneurysma arteriovenosum handeln. Alle übrigen klinischen Symptome, Veränderungen der Stärke des Pulses, Stauungserscheinungen, Drucksymptome, Neuralgien, Sensibilitätsstörungen wie Kribbeln und Ameisenlaufen, Lähmungen, Contracturen benachbarter Gelenke wurden vermißt. Ebenso fehlten fast völlig Störungen des Körperkreislaufes und der Herztätigkeit. Wenn auch keine Vergrößerung des Herzens und kein Herzfehler nachweisbar war, so bestand doch eine auffallend leichte reflektorische Beeinflußbarkeit des Herzens und des Gefäßsystems. Ob-

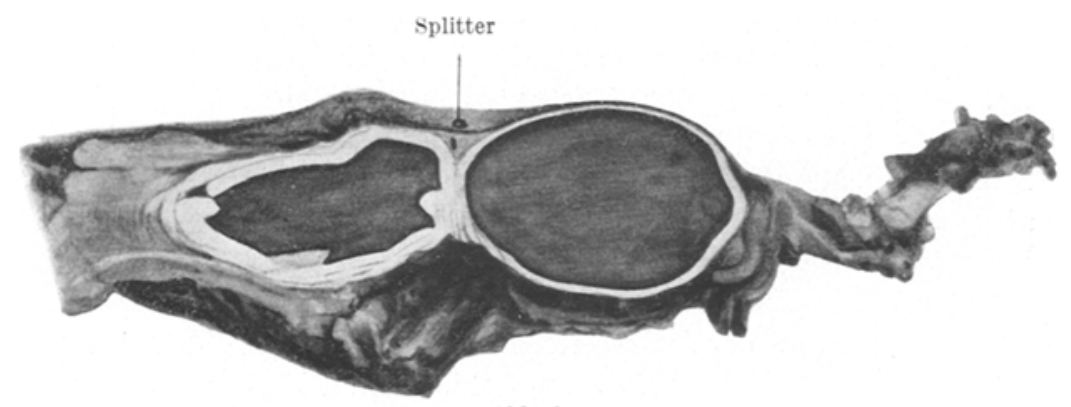

Abb. 3.

wohl das Aneurysma dem venösen Blute den Abfluß aus dem Uterus erschwert haben muß, fand sich keine eigentliche Staung.

Noch wichtiger sind die anatomischen Tatsachen. Bei der Operation selbst sowie unmittelbar nach Herausnahme des Tumors ließ sich feststellen, daß der kompressible Inhalt der beiden Gefäßauftreibungen aus einer flüssigen Masse, offenbar aus Blut bestand. Als nach der Härtung durch einen Horizontalschnitt die Kuppe des Tumors abgehoben war, lie $\beta$ sich erkennen, daß ausschließlich frisches Blut den Inhalt darstellte. Das nur durch die Konservierung geronnene Blut sa $B$ nirgends fest, es ließ sich fast in toto mit Leichtigkeit entfernen. Von einer Thrombosierung oder Schichtung oder Organisation des Gefäßinhaltes wurde nichts bemerkt.

Nach der Lage des einen sichtbaren Splitters in unmittelbarer Nachbarschaft der beiden Säcke wäre eine gleichzeitige Verletzung der Arterie and Vene so wenig ausgeschlossen wie eine doppelte Verletzung der Arterie selbst. Schon makroskopisch (Abb. 3) fällt sofort die verschiedene Dicke der Wand in den beiden Abschnitten des Aneurysmas auf. Die Wand des lateralen Sackes ist gleichmäßig dünn, die Wand des medialen Tumors ist gut doppelt so dick und verläuft mehr wellig. Derbes Bindegewebe schließt den dickwandigen medialen Teil ein. Man geht 
wohl nicht fehl in der Annahme, daß die Vermehrung und Wucherung des Bindegewebes einfach durch den chronisch mechanischen Reiz entstanden ist, welchen der pulsierende Abschnitt des Aneurysmas auf seine Umgebung ausüben mußte.

Der venöse laterale Abschnitt mehr herzwärts von dem medialen arteriellen Teil ist viel dünnwandiger. Aus der topographisehen Anordnung der beiden Abschnitte des Aneurysmas läßt sich die Saugwirkung, welche die Vene ausübt, gut verstehen. Durch die Saugwirkung des venösen Systems wird bei der Verletzung die Entstehung eines primären Hämatoms erschwert, gleichzeitig wird die Gefahr einer unmittelbaren akuten Verblutung dadurch ausgeschaltet.

Durch die traumatische Verbindung der Arterie mit der Vene ist die Vene im Laufe der Zeit umgebaut worden; sie ist makroskopisch arterialisiert. Trotz ihrer Erweiterung ist nämlich die Wanddicke der Vene noch ganz beträchtlich. Die überdehnte Venenwand ist jedenfalls stärker als die normale. Die verletzten Gefäßabschnitte der Vene haben sich ihrer neuen Aufgabe scheinbar nicht nur funktionell, sondern auch anatomisch angepaßt. Diesen Eindruck bekommt man unbedingt bei der makroskopischen Betrachtung,

Die mikroskopische Betrachtung lehrt uns aber eines anderen. Histologisch fällt an der Arterie selbst nichts Besonderes auf. Die Vene läßt überraschenderweise bei den verschiedensten Färbungen und auch bei der Elasticafärbung eine Veränderung der Wandstruktur vermissen. Die einzelnen Schichten sind getrennt nachweisbar, ihre Zusammensetzung weicht nicht von der Regel ab. Die Intima besteht nur aus Endothelzellen, elastische Bestandteile in Form einer eigenen Schicht oder eines Netzes sind nicht feststellbar. Die Media baut sich fast nur aus Bindegewebszellen auf, ebenso wie die Tunica externa. Obwohl hier das Aneurysma schon fast 5 Jahre bestand, so ist die Wand des venösen Sackes mikroskopisch fast nicht umgewandelt. Offenbar liegen hier beim Menschen die Verhältnisse doch anders wie bei der tierexperimentellen Transplantation eines Venenstücks in die Arterie. Stich und Zöppritz, Fischer und Schmieden fanden nämlich mikroskopisch bei ihren Experimenten eine ausgesprochene Arterialisierung der Venenwand.

Es geht ja aus den Abbildungen schon zur Genüge hervor, daß $\beta$ die erweiterten Gefäßabschnitte nur scheinbar nebeneinander in der gleichen Höhe liegen. Tatsächlich liegen sie in verschiedenen Ebenen hintereinander und besitzen nur in der Gegend der Fistel eine breitere Berührungsfläche.

Um den Verlauf des Blutstromes zeigen zu können, habe ich den arteriellen und venösen Abschnitt des Aneurysmas in einer Ebene liegend gezeichnet, und die Verhältnisse damit auf ein möglichst einfaches Schema gebracht $(\mathrm{Abb}, 4)$. Es ist daraus ohne weiteres ersichtlich, wie 
es in der Blutader zur Wirbelbildung kommen muß, wodurch die klinischen Symptome erklärt werden.

Die Pathogenese des Falles ist geklärt. Ein Splitter der Fliegerbombe drang wahrscheinlich durch das Foramen ischiadicum majus in die Tiefe und verletzte die Arteria und Vena uterina gleichzeitig. Die Vor* bedingungen sind günstig, 2 Venen begleiten meist die Arteria uterina. Die Gefäßwunden traten miteinander in direkte Verbindung, wohl begünstigt durch die Saugwirkung der Vene. Es kam zu einer Verklebung. Die Intima wuchs über die Wunde hinweg und so bildete sich eine direkte fistulöse Verbindung zwischen Arterie und Vene. Die Wirbelbewegungen des Blutes bedingen das besonders zentripetal fortgeleitete Geräusch, sowie die Erschütterungen der Gefäßwand und ihrer Umgebung, welche sich dem Tastsinn als zitternde Bewegungen oder als Schwirren verraten. Dabei möchte ich noch erwähnen, daß die Entstehung von Kriegsaneurysmen durch Fliegerbombensplitter nicht gerade häufig ist. Otto Hahn fand bei 122 Fällen aus Küttners Beobachtungsmaterial nur 3 Fälle durch Fliegerbombenverletzung entstanden.

Bei der Differentialdiagnose muß man in erster Linie denken an die abgesackten extraperitonealen Blutgeschwülste im Bereich des Beckenbindegewebes. Die Ligamenthämatome, welche nach $A . M^{2}{ }^{6}{ }^{6}$ ) durch direkte Verletzung, wie Pfählung, Kohabitation, Abtreibungsversuche, und nach indirekter Gewalteinwirkung, wie Körpererschütterung und schließlich spontan ohne Trauma zustande kommen können, sofern eine

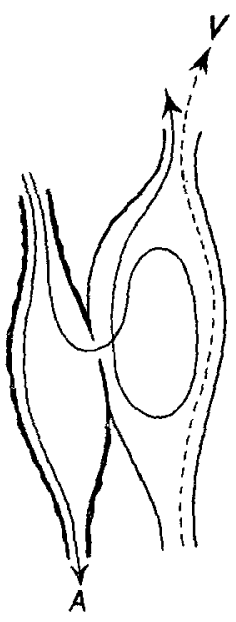

Abb. 4. örtliche oder eine allgemeine Disposition vorliegt, haben mit dem Aneurysma nur die einseitige Lage gemeinsam. Im frischen Zustande sind sie teils weich, allmählich nehmen sie eine härtere Konsistenz an. Beim Aneurysma läßt sich, wenigstens nach unserer Beobachtung, das Ligamentum sacrouterinum bei der rectalen Untersuchung isoliert heraustasten und umgreifen, beim Ligamenthämatom aber nicht immer.

Ferner kommen noch in Betracht: die einseitige, besonders deutlich fühlbare Pulsation der Arteria uterina bei einer Extrauteringravidität mit Haematocele peritubaria oder retrouterina. Ebenso ist es denkbar, daß Scheidencysten, welche als Abkömmlinge der Gartnerschen Gänge mit Vorliebe im Scheidengewölbe angetroffen werden, gelegentlich eine Scheinpulsation aufweisen, wenn sie der Arteria uterina unmittelbar aufliegen. Die tiefe Teilung der Aorta ist so selten, daß sie praktisch kaum zu erwägen ist, ebensowenig wie das pulsierende Sarkom. Bei der Differentialdiagnose muß man ausgiebig die rectale Untersuchung heranziehen, um die Lage, Größe, Ausdehnung der Geschwulst und ihre 
Beziehungen zum Uterus und zur seitlichen Beckenwand zu klären. Die Kompressibilität, welche allein den echten Blutgefäßgeschwülsten zukommt, ist überhaupt nur feststellbar durch die kombinierte Bauchdeckenscheiden-Mastdarmuntersuchung. So erwies sich bei unserer Beobachtung die Pulsation und das Schwirren bei der rectalen Exploration noch deutlicher fühlbar wie bei der vaginalen Untersuchung.

Eine falsche Diagnose könnte in einem solchen Falle von Aneurysma zu verhängnisvollen therapeutischen Irrtümern führen. Wird die Pulsation der Geschwulst nicht genügend beachtet und nicht kritisch verwertet und allein die Vorwölbung im Scheidengewölbe diagnostiziert, so könnte der parauterine pulsierende Tumor für ein dem Durchbruch naher akuter oder chronischer Absceß angesprochen werden. Schon bei der Probepunktion, erst recht bei der Eröffnung würde es zu einer verhängnisvollen Blutung kommen.

Erwähnenswert erscheint noch die Tatsache, daß das Aneurysma erst 5 Jahre nach der Verletzung Erscheinungen machte und hierauf festgestellt wurde. Bis dahin war es latent geblieben. Einen ähnlichen Fall mit langer Latenzzeit sah Hoffmann ${ }^{7}$ ). Erst $41 / 2$ Jahre nach der Verletzung trat das Aneurysma der Arteria und Vena femoralis im Adductorenkanal in Erseheinung.

Trotzdem dürfte die Anerkennung etwaiger Ansprüche an die Unfallversicherung oder Haftpflicht wegen Schädigung der Gesundheit oder wegen Gefährdung des Lebens im Zusammenhang mit dem Aneurysma nach dem gegebenen Tatbestande rechtlich auf keine Schwierigkeiten stoßen.

Das Aneurysma wurde in toto exstirpiert, ohne die normale Zirkulation wiederherzustellen. Unter Verzicht auf die konservierenden Operationsmethoden wählte man den einfachsten und sichersten Weg, zumal eine andere Methode überhaupt nicht in Betracht kam. Die Naht der Arterie und Vene wäre in dem kleinen, tiefen Wundgebiet ebenso unmöglich gewesen wie eine GefäBtransplantation. Außerdem braucht man auch, wie wir aus unseren täglichen operativen Erfahrungen mit der Radikaloperation der inneren Geschlechtsorgane wissen, bei der Ausschaltung des ganzen uterinen Gefäßgebietes durch die Exstirpation der Organe nicht mit Ernährungsstörungen zu rechnen, im Gegensatz zu den Unterbindungen bei Aneurysmen an den großen Gefäßstämmen der Extremitäten. Die unblutigen Behandlungsmethoden durch Kompression oder Gelatineinjektion mußten wir von vornherein ablehnen.

Es sind heute schon eine Reihe bestimmter Krankheitsbilder bekannt, welche man als Kriegsschädigungen der Frau oder gynäkologische Nachkrankheiten des Krieges aufzufassen pflegt. Ich nenne als indirekte nur die Zunahme der Hernien und Prolapse, worauf auch Piel ${ }^{8}$ ) aus unserer Klinik die Aufmerksamkeit gelenkt hat, die Zunahme der Ge- 
nach der Beobachtung eines Aneurysma arteriovenosum der Arteria usw. 139

schlechtskrankheiten, die Häufigkeit der Genitaltuberkulose und der kriminellen Aborte.

Im Rahmen der Lehre von den direkten Kriegsschädigungen der Frau als Folge der unmittelbaren Einwirkung von Kampfmitteln stellt der mitgeteilte Fall von Aneurysma arteriovenosum der Uteringefäße ein neues, bisher unbekanntes Krankheitsbild dar.

\section{Literatur.}

1) Mac Laren, Aneurysma of the internal iliaca. Ann. of surg. 1913, August; Amer. assoe. Number. Ref. Zentralbl. f. Chirurg. Nr. 40, S. 1726. $-{ }^{2}$ ) Colmers, Das Aneurysma der Arteria obturatoria infolge von Schußverletzung und seine Bedeutung. Zentralbl. f. Chirurg. 1917, Nr. 50. - ${ }^{3}$ ) Siehe Otto Hahn, Bruns' Beitr. z. klin. Chirurg. 124, Heft. 2. Die Kriegsverletzungen der Blutgefäße. (Auf Grund des von Küttner im Welthriege beobachteten Materials.) - ${ }^{4}$ ) Siehe $R$. Stich und H. Fromme, Die Verletzungen der Blutgefäße und deren Folgezustände (Aneurysmen). Ergebn. d. Chirurg. u. Orthopäd. 13. - $\left.{ }^{5}\right) v$. Gaza, Grundriß der Wundversorgung und Wundbehandlung. Berlin 1921. - $\left.{ }^{6}\right)$ A. Mayer, Die Unfallerkrankungen in der Geburtshilfe und Gynäkologie. F. Enke, Stuttgart 1917. - 7) Hoffmann, Ein $4^{1} / 2$ Jahre nach der Schußverletzung in die Erscheinung tretendes Aneurysma der Arteria und Vena femoralis im Adductorenkanal. Dtsch. med. Wochenschr. 1918, S. 1079. - $\left.{ }^{8}\right)$ P. Piel, Über Genitalprolapse und Unterleibsbrüche im. Kriege. Zentralbl. f. Gynäkol. 1918, S. 237. 